\title{
Noción de frontera y objetos de cooperación en escenarios de diferencia epistemológica
}

\section{Boundary notions and objects of cooperation in contexts of epistemological difference}

Jorge E. Vergara-Vidal (jorge.vergaravi@uv.cl) Escuela de Sociología, Universidad de Valparaíso (Valparaíso, Chile) https://orcid.org/0000-0002-7712-4090

Octavio Avendaño-Pavéz (oavendan@uchile.cl) Departamento de Sociología, Universidad de Chile (Santiago, Chile) https://orcid.org/0000-0001-6945-5327

\begin{abstract}
This paper examines the uses of the notion of boundary in sociological theory as a useful analogy to describe spatiality in situations of material semantic exchange and cooperation between different actors and communities. These uses do not account for a limit or a form defined by its location between the borders of different territories, but for a relational space that translates and symmetrizes the epistemological differences of the actors, and whose object quality allows for heterogeneous localization. Finally, we evaluate the extent to which the objective condition of boundaries allows cooperation practices to be scenarios of epistemological difference and its effect on the cohesion between actors and communities defined by it.
\end{abstract}

Key words: boundary, communities of practice, boundary object, intermediary objects.

\section{Resumen}

Este artículo explora los usos de la noción de frontera en la teoría sociológica como una analogía útil para describir la espacialidad en las situaciones de intercambio y cooperación semántico material entre actores y comunidades diferentes. Esto usos no dan cuenta de un linde o de una forma definida por su ubicación entre los bordes de territorios distintos, sino de un espacio relacional que traduce y simetriza las diferencias epistemológicas de los actores y cuya cualidad objetual permite una localización heterogénea. Finalmente, se evalúan los alcances que la condición objetual de las fronteras permite a las prácticas de cooperación en escenarios de diferencia epistemológica y su efecto en la cohesión entre actores y comunidades definidos por ésta.

Palabras clave: frontera, comunidades de prácticas, objeto frontera, objetos intermediarios. 


\section{Introducción}

La noción de frontera ha sido utilizada en las ciencias sociales para describir tanto una configuración espacial intermedia, o común, entre dos formas y/o colectivos sociales, como una cualidad demarcatoria de los objetos, expresada en los bordes o en los límites de un espacio (Lamont y Molnár The study of boundaries in the social sciences, Jirón Urban border). Ambos usos no son excluyentes, sin embargo, tienen un efecto epistemológico diferente según se quiera entender la frontera como un espacio o como un objeto. El presente texto explora la forma en que es abordada la noción de frontera en la obra de Georg Simmel (1858-1918), de Robert Ezra Park (1864-1944) y de Susan Leigh Star (1954-2010), con el fin de exponer cómo esta consecución de reflexiones, inspiradas unas en otras, dan forma a una noción de frontera en la que un objeto actúa como un espacio. Esta particular semiótica material (Law Material semiotics) otorga un específico sentido relacional a objetos espaciales heterogéneos. Se trata, como sostiene Susan Leigh Star (This is not a boundary object), de objetos difusos, vagos, pero suficientemente buenos para sostener la cooperación entre comunidades de prácticas diferentes, en especial por ser capaces de articular o dar cuenta de una posibilidad de frontera entre éstas.

Esta noción objetual de frontera da cuenta de ecologías donde se suspenden eventuales conflictos de intereses y se articulan arreglos que permiten que grupos diferentes trabajen juntos sin consenso. Intermedian el hacer de los actores en un sentido material, informacional y pragmático, al punto que pueden ser vistas como actos en común de éstos. Situaciones similares son abordadas en los trabajos de Michel Callon (Some elements of a sociology of translation) y Dominque Vinck (De l'objet intermédiaire à l'objet-frontière), que tienen en común observar objetos materiales que afectan la coordinación de la acción en situaciones donde los actores presentes provienen de mundos de la vida diferentes (Akrich et al. Sociologie de la traduction).

En la primera parte, el texto expone cómo esta noción de frontera toma cuerpo en las reflexiones de Simmel y de Park en torno a la idea de entidades y espacios donde culturas y/o asociaciones diferentes se entrelazan. En la segunda parte, se aborda la forma como (a partir del trabajo de Park, Star y otros) desarrollan la noción de objeto frontera como un instrumento para describir y agrupar a diversos objetos que cumplían con el trabajo epistemológico de sostener la cooperación entre comunidades de prácticas diferentes. En la tercera parte, se compara esta dimensión práctica de la espacialidad de frontera con el trabajo de traducción y con la noción de intermediación mediada por objetos y se discute la complementariedad entre las nociones de traducción, intermediación y frontera. Finalmente, se evalúan los alcances que la condición objetual de las fronteras permite a las prácticas de cooperación en escenarios de diferencia epistemológica y su efecto en la cohesión entre actores y comunidades definidos por ésta.

\section{Simmel, Park y una sociología de las fronteras}

El Extranjero es un texto que ocupa un lugar especial dentro de la obra de Simmel (Sociología), en particular porque se utiliza una noción distinta de la que venía trabajando para caracterizar las fronteras como fenómenos sociales. En trabajos anteriores, Simmel utilizaba la noción de frontera como una línea demarcatoria (Begrenzung) que era expresión del conflicto de intereses entre dos grupos o esferas de la vida personal (The social boundary). 
En El Extranjero, presentado como un excurso dentro de las formas de socialización, describe una forma especial de relación recíproca entre entramados de prácticas aparentemente opuestos y distantes que son reconciliados en un límite ambivalente. El extranjero descrito por Simmel no es un nómada, es una figura capaz de involucrarse en redes de vínculos que no le son ni totalmente propias ni totalmente ajenas (May y Lahad The involved observer). Es un observador comprometido con lo que sucede, tanto dentro como fuera de la situación socioespacial en que se ve involucrado, y en la que no es una entidad meramente migrante o pasajera, sino expectante y especulativa (Möllering The nature of trust; Southerton Boundaries of the 'us' and 'them'). El extranjero es una figura relativista, que inaugura una perspectiva relacional de la modernidad en que las interacciones no son explicadas por la esencia de las cosas sino como un efecto de éstas (Robles La ambivalencia como categoría sociológica en Simmel; Karakayali Two ontological orientations in sociology).

Simmel, fiel a su método, no ahorra analogías en su digresión para describir el espacio que, como efecto de los mundos que carga, el extranjero puede ocupar. Le describe como un intermediario, como un comerciante, como efecto del intercambio entre dos economías. Así es capaz de crear múltiples combinaciones, ampliaciones y fuentes con tal de mantener los dispares mundos económicos unidos. El comercio siempre puede emplear más personas que la producción primaria, por tanto, el extranjero llegará a los lugares de intercambio como quien llega a un espacio natural. No es un propietario territorial, pero a cambio es móvil, ocasional y movible. A su vez, como efecto de no estar unido fuertemente a los grupos o a sus tendencias, parece estar equidistante de ellas, tener la posibilidad de ser objetivo, neutro o flexible dada la libertad que le dan los vínculos de los que carece. Eso también le permite ocupar y transitar por "boundary places [...], espacios entre dos o más marcas [...] en que los hombres pueden encontrarse para el cambio de mercancías, si no en términos amistosos, al menos sin hostilidades" (Simmel 2015:613).

Simmel describe estos espacios de frontera como esferas de trato social donde ocurre un "contacto objetivo" entre comunidades diferentes o adversarias, y donde es posible la cooperación o el intercambio entre ellas, sin que cese la diferencia o la hostilidad, una zona en que los actores salen de su territorio sin entrar en el de otros, un espacio vacío que "pasa a ser considerado como depósito y expresión de la acción recíproca sociológica" (Simmel 2015:613). Tanto el espacio de frontera como el extranjero son definidos por Simmel como objetos neutrales que hacen posible el intercambio de objetos simetrizados como equivalencias por la operación comercial, un modo de cooperación que no suspende la hostilidad o la negociación, pero no la requiere para funcionar.

El extranjero, para Simmel, no solo es un actor dentro de estos espacios de frontera: también los lleva consigo, los encarna y es parte de su objetividad (Mol Not quite clean). Robert Ezra Park (Human migration and the marginal man) integrará estos aspectos en una versión más ecológica radicada en lo que denominó el Hombre Marginal, una forma de caracterizar la condición fronteriza y ambigua de la persona migrante como constitutiva de bordes que enlazan sociedades físicamente distantes. Es lo que Park sugiere que ocurre en la mente de la persona migrante, donde ésta adapta e integra su identidad en un espacio donde se tocan sus culturas de origen y de llegada. Esto le otorga a su identidad cierta hibridez y también cierta capacidad de sincretismo, pues el margen en el que habita y que articula nunca integra o fusiona completamente los dos mundos diferentes que reconoce y enlaza. 
El hombre marginal por excelencia es el extranjero de Georg Simmel, sostiene Park y es en sus espacios interiores, particularmente en su mente, donde se articula una esfera de trato social, una "donde se producen los cambios y fusiones de la cultura, donde mejor podemos estudiar los procesos de civilización y de progreso" (Park 1928:893). La mente del hombre marginal es caracterizada de manera similar al espacio de frontera de Simmel, salvo que, en esta ocasión, el espacio no es del todo neutral, sino que está supeditado a las tareas de adaptación de la asociación que lo articula y que lo contiene. La reflexión de Simmel y la de Park están unidas por una concepción espacial de objetos donde son posibles los arreglos o tratos de intercambio, las mezclas y las fusiones entre colectivos, culturas o comunidades diferentes.

Dada la forma como ambos autores caracterizan a estos espacios, los márgenes entre dos o más mundos sociales son heterogéneos, flexibles y objetuales, pero además se articulan no como lindes, sino como espacios donde se organizan el intercambio y la cooperación entre colectivos diferentes. Es esto lo que está en el centro de la sociología de frontera de Simmel y Park: un objeto de cooperación inesperado y espacial donde operaciones de intercambio y traducción pueden ser realizados.

La influencia del hombre marginal de Park, y por defecto la de Simmel, es central para la forma como Star y Greisemer (Institutional ecology, translations and boundary bbjects), organizaron la arquitectura del objeto frontera. Separadamente, Star (This is not a boundary object) y Griesemer (Sharing spaces, crossing boundaries) reconocen haber considerado inicialmente la noción de marginalidad utilizada por Park para caracterizar a estos objetos, compartidos como espacios por dos culturas de prácticas diferentes. Fue la posibilidad de que la noción de marginalidad se entienda erradamente lo que convenció a Star y a Griesemer de desestimarla a favor de la noción de frontera.

Al observar lo que ocurría con el espacio del Museo de Zoología de Vertebrados de la Universidad de California en Berkeley, donde las relaciones que los científicos establecían con los mundos de la vida externos (sus visitantes y sus proveedores de animales e insumos) estaban plagadas de objetos que permitían a éstos mundos enlazarse unos con otros (bound), notaron que los carteles, formularios y estandarizaciones también dibujaban espacios relacionales que permitían el intercambio y la cooperación entre los actores, sin que las diferencias entre ellos y sus mundos se desvaneciera. Consideraron que la metáfora de marginalidad de Park podía ser malinterpretada. Podía llevar a pensar que estos fenómenos se producían en los bordes de los mundos y, a su vez, considerar que éstos tenían centros y periferias, cuando en realidad lo que estaban describiendo, como Simmel, era un espacio de enlace, un nodo de intercambio y cooperación entre mundos sociales o comunidades de prácticas diferentes.

\section{Star, Greiseimer y el objeto frontera}

En trabajos anteriores, Star (Simplification in scientific work, The sociology of science and technology) había estudiado objetos que aportaban predictibilidad a los actores en la medida que articulaban diferencias y límites dentro de las situaciones de interacción. Estos objetos resultaban útiles también para enlazar los elementos diferentes, produciendo cohesión entre ellos y operando, a la vez, como límites y nodos. Pero fue en un texto sobre la inteligencia artificial (The structure of ill-structured solutions) que arriesgó un concepto que utilizaría para describir lo observado en el museo de Berkeley: objetos frontera (boundary objects). 
Los definió como objetos "lo suficientemente plásticos como para ser adaptables a través de múltiples puntos de vista, pero que mantienen la continuidad de la identidad" (Star 1989:37) y propuso cuatro tipos de éstos: repositorios, tipos ideales, terrenos con límites coincidentes y formas estandarizadas. Luego, en su trabajo sobre el Museo de Zoología de Vertebrados, Star, junto a Griesemer (Institutional ecology, translations and boundary objects) profundizaron en los aspectos que caracterizaban a los objetos frontera observados (flexibilidad interpretativa, estructura material, organizativa y granularidad) llegando a la conclusión de que constituyen una suerte de arreglos que permiten que diferentes grupos trabajen juntos sin consenso y cuyas formas posibles no son arbitrarias. Son esencialmente infraestructuras orgánicas surgidas de necesidades de información y trabajo, percibidas localmente por grupos que desean cooperar (Star This is not a boundary object).

Lo importante para los objetos frontera, sostienen Star y Griesemer (Institutional ecology, translations and boundary objects), es que evidencian cómo la estructura de las prácticas y el lenguaje expresan materialmente formas de hacer cooperativo y, en esa línea, propusieron cuatro modos de emergencia, aunque sus posibilidades materiales no quedan agotadas en ellas:

Los "repositorios u objetos modulares", como las bibliotecas o los museos, donde personas de diferentes mundos pueden usar o pedir prestados objetos indexados de una forma estandarizada para sus propios fines, sin tener que negociar directamente las diferencias de propósito y sin exponerse a los problemas de heterogeneidad causados por las diferencias entre los objetos o unidades indexadas.

Los "tipos ideales", que corresponden a objetos como los diagramas, los atlas u otro tipo de representaciones descriptivas que, en realidad, no describen con precisión los detalles de ninguna cosa o localidad, pero son suficientes para dar una idea de ello, pues son abstracciones y, por lo mismo, son bastante vagos, pero precisamente por eso son muy adaptables localmente, siendo útiles como medios de comunicación y cooperación simbólica, como una hoja de ruta "suficientemente buena" para cualquier lugar. Un ejemplo de un tipo ideal es la especie. Este es un concepto que, de hecho, no describía ningún espécimen, que incorporaba datos concretos y teóricos y que servía como medio de comunicación entre ambos mundos. Los tipos ideales surgen con diferencias en el grado de abstracción. Funcionan para la eliminación de las contingencias locales del objeto común y tienen la ventaja de la adaptabilidad.

Los "límites o los contornos coincidentes", que son objetos comunes que comparten contornos, pero tienen diferentes contenidos internos. Surgen en presencia de diferentes medios para agregar datos y cuando el trabajo se distribuye en un área geográfica de gran escala. El resultado es que el trabajo en diferentes sitios y con diferentes perspectivas se puede realizar de manera autónoma, mientras que las partes que cooperan comparten un referente común. La ventaja es la resolución de diferentes objetivos bajo un concepto de diferencia ecológica

$Y$, finalmente, los "formularios estandarizados", que son objetos frontera diseñados como métodos de comunicación regular y estandarizada entre grupos de trabajo dispersos. Los resultados de este tipo de objeto de frontera son indexaciones o colecciones estandarizadas que coinciden con lo que Latour denomina "móviles inmutables", esto es, objetos que se pueden transportar a una gran 
distancia y transmitir información inmutable. Las ventajas de este tipo de objetos son que se eliminan las incertidumbres locales, por ejemplo, en la recolección de especies animales.

Para Star (This is not a boundary object), los objetos frontera surgen a lo largo del tiempo de la cooperación duradera entre comunidades de prácticas, en tanto acuerdos de funcionamiento que resuelven anomalías de naturalización sin imponer la naturalización de una comunidad o del exterior. Su construcción en un fenómeno propio de las comunidades y de sus distinciones ecológicas, por lo cual su construcción depende de actores con, a lo menos, dos puntos de vista diferentes. Los objetos frontera son utilizados por las comunidades de prácticas para resolver problemas relacionados con la distribución de elementos de conocimiento y la heterogeneidad epistemológica de los actores. En específico, organizan un nodo o un espacio en común con otras comunidades con las que desean cooperar. En el caso del Museo de Zoología de Vertebrados, donde se estudió los modos como los científicos del museo articulaban la cooperación con sus proveedores, los objetos frontera se multiplican cada vez que una comunidad de práctica se topa con otras o surgen nuevas heterogeneidades.

Como esto ocurre constantemente, los límites que denotan los objetos frontera se muestran tan cambiantes como persistentes, como ocurría con los modelos y métodos de recolección y curatoría que utilizaba el museo. En el caso del Museo de Zoología de Vertebrados, éstos databan de más de un siglo y ordenaban, en lo grueso, la historia natural de California según la relación entre topografía y especies no humanas que había elaborado su primer director, Joseph Grinnell, de modo que los taxones y las formas de presentación de las especias no habían tenido cambios desde 1908. En cambio, los formularios de ingreso, los manuales de prácticas y procedimientos que relacionaban a científicos y tramperos, quienes proveían de animales para las muestras del museo, sí habían tenido cambios desde la época de Grinnell.

Los objetos frontera no emergen solo para denotar distinciones entre diversos mundos sociales o comunidades de prácticas, sino sobre todo sitúan o localizan a los actores en una espacialidad común, una forma de orden métrico y accional o una estandarización de las prácticas. Dentro de esta operación forman parte de configuraciones de sentido mayores, de semióticas materiales dispuestas para producir cuantificaciones, órdenes y distinciones flexibles y funcionales a situaciones llena de heterogeneidad. Colaboran decididamente con la puesta en escena del mismo espacio de frontera que demarcan y articulan. Por lo mismo, los objetos frontera no emergen aisladamente, y sus combinaciones y superposiciones dan pie a conjuntos orgánicos que corresponden a lo que Star (This is not a boundary object) denomina infraestructuras frontera.

Tal como ocurre con una carretera, un puente o una red de cañerías, los objetos frontera se basan en la acción, particularmente en el trabajo, y cooperan para que determinadas prácticas ocurran, para que enlazamientos entre actores y comunidades de prácticas sucedan y para que cierto tipo de cohesión (cooperación) se enacte entre ellos. En tal sentido están sujetos a la reflexión y a la adaptación local, a su vez que son distribuidos a través de todas las dimensiones de la acción, es decir, son n-dimensionales (Bowker y Star Work and infrastructure).

Esta adaptación local requiere que las comunidades de prácticas mantengan la identidad del objeto frontera tan vaga como la de un objeto común, y que a la vez sea específica a su uso local, en ese mundo social en particular. Cuando la distancia entre esas dos formas del objeto se amplía o se 
estandariza, los objetos frontera comienzan a moverse y cambiar a infraestructuras, a estándares metodológicos, a prototipos, a tipologías y a otros procesos.

Dado que reside epistemológicamente entre los mundos sociales o entre las comunidades de prácticas, el objeto frontera siempre está mal estructurado, no puede ser otra cosa que un arreglo temporal y heterogéneo (flexible interpretativamente), aún en una escala combinada, estandarizada o infraestructural. Sin embargo, su capacidad de empaquetar y desplazar enlaces relacionales a través de arreglos de cooperación, informáticos y pragmáticos menos locales, como estándares y tipologías, le permite desplegar con mayor claridad su condición infraestructural. De esta manera, conjuntos o combinaciones de objetos frontera no solo colaboran con prácticas más complejas, sino también con asociaciones más amplias y más densamente pobladas de actores diversos o de comunidades de prácticas diferentes.

Las infraestructuras, que responden a tipologías y que requieren para su conformación de elementos prototipados, estandarizados y enlazables masivamente entre sí, se desarrollan mediante estos sistemas de objetos frontera que, como se ha dicho, colaboran con el trabajo cooperativo sin consensos. La relación con los consensos entre actores, y que es parte de la flexibilidad interpretativa que los caracteriza, es uno de los aspectos que son posibles de observar en los objetos frontera de manera independiente de su tamaño o a la densidad de las asociaciones a las que están vinculados. En el caso de los objetos frontera infraestructurales, esto puede ser aún más claro, sobre todo porque la cantidad de mundo sociales que enlazan es mayor y no están solo acotados a actores humanos.

Star y Griesemer (Institutional ecology, translations and boundary objects) sostienen que el modelo objeto frontera aborda el problema de las representaciones comunes en la intersección de diferentes mundos sociales de una manera distinta a la adoptadas, que denominan el modelo de interesamiento de Latour, Callon y Law. En particular mencionan los trabajos de Bruno Latour sobre los estudios de laboratorio, de John Law sobre las naves portuguesas y el control a distancia, y en especial Michel Callon (Some elements of a sociology of translation) en su observación sobre los científicos y pescadores en la Bahía de Saint Breuic, de los que rescataron las nociones de "acción a distancia" y "traducción", para contrastar la dinámica en que se daba en la cooperación entre mundos sociales o comunidades de prácticas diferentes. Por su parte, Dominque Vinck (De l'objet intermédiaire à l'objet-frontière) ha sostenido que ambos modelos pueden ser complementarios, lo que obliga a las posibilidades de una noción ampliada de la noción de frontera, esta vez ya no como objeto o espacio, sino como práctica, o como política, tal como lo propone el modelo de traducción de Callon.

\section{La noción de frontera como un modo de involucrarse con el mundo}

La noción de traducción de Michel Callon (Some elements of a sociology of translation) fue expuesta como un marco analítico para el estudio del papel que juegan la tecnología y la ciencia en la configuración de las relaciones de poder. Esta fue aplicada a un caso en particular en que actores humanos debían entrar en relación con actores no humanos para la introducción de jaulas de producción de vieiras en la bahía de Saint Brieuc. Callon propone estudiar las relaciones de poder, en primer lugar, considerando en el análisis a todos los actores involucrados en éstas, independiente de su condición humana, no humana, técnica, social o natural y, en segundo lugar, dando igual valor 
a estos actores movilizados, tornando simétricas las explicaciones y cualidades que se imputan a ellos.

La traducción describe las operaciones que pueden ser observadas como resultado de aplicar estas premisas que Callon, además, articula como radicalización de lo propuesto por el Programa Fuerte para la Sociología del Conocimiento planteado por David Bloor y sus colegas de la Universidad de Edimburgo. Para Callon, "la traducción es el mecanismo por el cual los mundos social y natural toman forma progresivamente [y su] resultado es una situación en la cual ciertas entidades controlan otras" (Callon 1984:278). Tal mecanismo contiene cuatro tipos de movimientos simultáneos que el autor caracteriza con preguntas: ¿cómo resultar indispensable? (la problematización), ¿cómo mantener a los aliados en su sitio? (el interesamiento), ¿cómo definir y coordinar los roles? (el enrolamiento) y ¿cómo movilizar a los aliados? (la representatividad de sus voceros). Al traducir, los actores deben "expresar en el propio lenguaje lo que otros dicen y quieren, por qué actúan como lo hacen y cómo se asocian entre sí" (Callon 1984:278), por ello para Callon "traducir es desplazar".

En el caso descrito, un grupo de científicos interesados en la introducción en Francia del método de cría protegida de vieiras mediante jaulas, deben ser capaces de traducir los intereses divergentes de pescadores, otros científicos, jaulas y vieiras en la bahía de Saint Brieuc, de manera que, sin volverse similares, se vuelvan coherentes entre sí, es decir, que puedan enlazarse, asociarse y funcionar juntos. Para lograr esto los científicos configuran lo que Callon llama un punto de paso obligado (PPO), ubicado en un problema o pregunta que todos los actores debieran tener interés en resolver y que, por tanto, podría movilizarlos y hacerlos confluir. En el caso observado, el punto de paso obligado radica en los dispositivos (colectores) que los científicos proponen para la crianza de las vieiras, por lo que debieran ser capaces de responder preguntas relacionadas con los intereses de los actores involucrados, como: ¿se fijarán solas a ellos las vieiras?, ¿a qué metas se puede aspirar utilizando estas jaulas?, ¿qué podemos aprender las vieiras a partir de estos dispositivos?, entre otros.

Callon (Some elements of a sociology of translation) propone a las vieiras como actores que también deben abordar el punto de paso obligado. ¿Será de utilidad para perpetuarnos? Se imagina que han de preguntarse al observar y decidir entrar en asociación con las jaulas colectoras y, a través de ellas con las otras entidades involucradas en la traducción, pescadores, científicos y comunidades de conocimiento. John Law sostiene que "traducir es conectar, es desplazar, mover, cambiar de lugar, una modalidad, una forma, otra manera de conservar algo" (Law 2002:99) y, en tal sentido, el punto de paso obligado logra motivar tales acciones.

La idea de Callon de un punto de paso obligado, implica que todos los actores han de concurrir a ese espacio frontera, pero la propuesta de Star es que el objeto frontera no solo configura un destino obligado para los actores sino, antes que nada, una ecología común de contenido material, informacional y pragmáticamente en un objeto. La idea tiene semejanzas con otras figuras pragmáticas, como las zonas de operación de Schutz y Luckmann o los centros de cálculo de John Law, que son espacialidades donde se configuran traducciones y movilizaciones de actores, pero a diferencia de ellas, un objeto de frontera dista de ser un espacio de debate, donde hay presencia de una autoridad sustantiva o metodológica (Star y Gresiemer Institutional ecology, translations and 


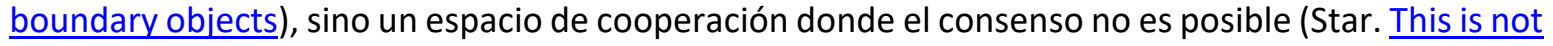
a boundary object).

Una de las innovaciones del modelo es que buena parte de la configuración del espacio de interacción emerge de la condición de frontera del objeto, de modo que el efecto de este sobre la situación es estructurante y no es el mero resultado de esta, como podría entenderse a partir de lo propuesto por el modelo de interesamiento y traducción, donde los objetos son el efecto de las interacciones que los incluyen. Los objetos frontera, y esto es recogido también por Trompette y Vinck (Retour sur la notion d'objet-frontière), contienen una capacidad performativa que es a la vez una capacidad infraestructural. Al enlazar mundos sociales o comunidades de prácticas diferentes, los objetos frontera les dan soporte espacial a prácticas específicas (de cooperación sin consenso) y actúan orgánicamente como infraestructura, sobre todo al constituir conjuntos y combinaciones, como sostienen Bowker y Star (Work and infrastructure).

Para Star y Griesemer (Institutional ecology, translations and boundary objects) las dinámicas involucradas en la noción de los objetos frontera demuestran que estos responden a un modelo relacional diferente al modelo de interesamiento y traducción que asocian a Latour, Callon y Law, donde ocurren desplazamientos y negociaciones. El modelo de objetos frontera, como afirma Star y corrobora Meloni (From boundary-work to boundary object) está basado en una noción espacial de trabajo agenciado materialmente en un objeto, que ayuda a describir lo que ocurre en las ecologías relacionales, donde las acciones de trabajo y cooperación ocurren en torno a acuerdos de trabajo y heterogeneidad.

De esta manera, los objetos frontera en tanto formas particulares de acción y cooperación, no solo sirven para indicar los límites de un mundo de la vida respecto de otro, determinando espacios centrales y periféricos como propone Leandro Rodríguez sino, sobre todo, para enlazar mundos de la vida entre sí, permitiendo la interacción y el desplazamiento de actores entre ellos. Operan de una manera extendida, lo que podría ocurrir con un punto de paso obligado, si la operación de este no implicara solo hacer coincidir a comunidades de prácticas en una dirección común, sino permitir el paso, en ambos sentidos, de actores entre las mismas comunidades u otras (Contu On boundaries and difference, Hawkins, Pye y Correia Boundary objects, power, and learning). Expresan lo que Laurent Thévenot denomina "modos de involucramiento con el mundo" (La acción en plural).

Para Thévenot las figuras accionales con las que los enfoques interaccionistas y sistémicos enlazan a los individuos con los sistemas técnicos (individualidades decisoras, negociadoras o calculadoras) dejan de lado los modos específicos y colectivos en que esto se produce, de modo que solo permiten apreciar justificaciones parciales de la acción, pero no el conjunto de las operaciones involucradas en esta. En tal sentido, los objetos frontera son parte del equipamiento socialmente dispuesto para producir los enlazamientos materiales, por los que la acción se hace viable y resulta sobre todo útiles "para la coordinación de los seres humanos dentro de un mundo material" (Thévenot 2016:68).

Para Dominique Vinck (De l'objet intermédiaire à l'objet-frontière), objetos que intermedian y enmarcan la acción como textos (correos, informes, documentación técnica, formularios en blanco y completos, etc.), instrumentos, insumos, animales, plataformas, entre otros, pueden ser equipados como objetos de frontera si, por ejemplo, se agrega a estos objetos cualquier elemento que permita vincularlos con soportes convencionales y espacios de circulación. Por ejemplo, una 
etiqueta pegada a un vaso de muestra biológica, a una caja de electrodoméstico, u otro que contenga metadatos que permitan inscribir al objeto en un conjunto más grande.

Esta posibilidad es interesante, pues implica que cualquier objeto puede ser enlazado a un conjunto infraestructural por medio de datos de coordinación tan simples de integrar como las etiquetas o, dicho de otro modo, las señas de identidad que implican la cuantificación y ordenamiento adscripto de las entidades son parte de los conectores que permiten organizar y coordinar colectivos e infraestructuras.

El aporte específico de la noción de objeto frontera radica en enfatizar la presencia de coordinaciones en escenarios donde no es posible el debate que lleva al consenso, alojadas en formatos materiales. Su heterogeneidad epistemológica y su flexibilidad interpretativa no son simples cualidades, sino condiciones prácticas de tales funciones, por tanto, no todos los objetos capaces de intermediar la acción corresponden a objetos frontera. Tampoco serían el equipamiento epistemológico lo que lo constituye en tal, puesto que ello cerraría la posibilidad del objeto de ser flexible interpretativamente, enlazándolo a una comunidad de prácticas determinada, más que dotándolo de la posibilidad de articularse como espacio común entre comunidades diferentes.

Con todo, la propuesta de equipar a un objeto intermediario como objeto de frontera alude a un aspecto que resulta central para entender el fenómeno y que está vinculado a la noción de "trabajo". El "trabajo de equipamiento" propuesto por Vinck implica una "actividad colectiva que consiste en acordar los elementos que deben agregarse a los objetos intermediarios para que encajen en un espacio de intercambio entre actores más o menos heterogéneo" (Vinck 2009:66), lo cual toca dos puntos claves relacionados con los espacios frontera. Primero, que el trabajo que despliegan contiene un proceso de diseño y elaboración de un objeto material y, segundo, que ello es orientado por la condición ecológica y situada del objeto, en este caso, una frontera, un espacio común.

\section{Conclusión. La noción de frontera como espacio común}

Como ha sido posible apreciar en lo anterior, la noción frontera utilizada por Simmel, Park y Star decanta progresivamente hacia una concepción del espacio como un elemento heterogéneo y en su empleo como técnica de lo común a través de su objetualización simbólica y material. Ello explica que el camino que une las analogías de Simmel con las metáforas de Star, no termine en figuras inmateriales, sino en objetos realmente existentes. Se trata de una noción de frontera que, para referirse a un espacio compartido, donde el sentido del allí y el acá se confunden, recurre a desarrollar el trabajo epistemológico de agenciarla y movilizarla por y a través de objetos.

De esta manera, la noción de frontera aquí observada no solo parte de las situaciones de coordinación, sino que también participa de ellas como un tipo específico de prácticas materiales. Eso le permite ser explicada, observada y registrada a través de la metáfora de un objeto que la encarna y que enlaza actores, comunidades y técnicos diferentes, sin la necesidad de oponerlos como hacen las metáforas basadas en la divergencia de intereses. Tal como el hombre marginal, el objeto frontera pertenece a más de una cultura o a más de un mundo social al mismo tiempo, es híbrido porque emerge creado por la interacción de las partes heterogéneas que lo componen (Callon y Law. Agency and the hybrid collectif), habita en un mundo incierto, por lo cual tiene la 
necesidad de adecuarse situadamente, lo que abre la posibilidad de trazar local y temporalmente los arreglos empleados en esto y, obviamente, responde a una existencia material.

Si la flexibilidad interpretativa del espacio de frontera estaba ya presente en Simmel, y en Park se denotaba la modularidad propia de la localización múltiple del hombre marginal, el aporte de Star, aún antes de su colaboración con Griesemer, radica en sumar a lo anterior la condición material. Con ello se suman las tres características de los objetos frontera señalados por Star: su flexibilidad interpretativa, su modularidad y su materialidad. Estos tres aspectos son claves para establecer la escala y el alcance con que actúan y que los diferencian de otros objetos intermediadores (Trompette y Vinck. De l'objet intermédiaire à l'objet-frontière) debido a la posibilidad de operar infraestructuralmente sobre la acción.

Dominique Vinck (El papel de los objetos intermediadores en la interdisciplinariedad) aprecia algo similar en objetos que habilitan prácticas de coordinación como textos, instrumentos, insumos, animales, plataformas, entre otros. Pero si bien los objetos materiales que intermedian las acciones de coordinación pueden resultar útiles para abordar aspectos relacionados con los procesos de diseño y elaboración de objetos e infraestructuras frontera, es importante notar que estos tienden a ser específicos a ciertos grupos y no comunes necesariamente a ellos, como ocurre con los textos escritos bajo lenguajes particulares, los instrumentos técnicos, las representaciones gráficas o los formularios técnicos, entre otros.

Del mismo modo, la noción de frontera no es equivalente a la noción de intermediación. Aun cuando esta última, debido a su capacidad de encuadrar la acción, puede resultar útil para identificar y calificar relaciones, actores, cursos de acción, redes, procesos, organizaciones y actividades relacionadas, no necesariamente lo es para describir aquello que permite que diferentes grupos trabajen juntos sin consenso. Tanto este aspecto, como los asociados a su escala y al alcance de su incidencia, son claves para determinar que la noción de frontera empleada no es puramente posicional, sino que tiene que ver con las prácticas organizativas que se ayuda a desplegar. Las formas que asumen los objetos frontera no son arbitrarias y ello permite que el modelo no sea extendido al conjunto de los objetos materiales. Los objetos frontera responden a necesidades comunes de grupos diferentes. Se articulan como infraestructuras orgánicas que emergen de requerimientos de información y trabajo configurados en situaciones cooperativas. Constituyen estructuras de prácticas y lenguaje necesarias para que actores y comunidades de prácticas diferentes pueden cooperar y hacer cosas juntos en escenarios de divergencia epistemológica.

En todo ello, la noción sociológica de frontera como un objeto práctico instalada por Simmel y consolidada por Park resulta fundamental, pues releva la utilidad de la figura de frontera para evidenciar la flexibilidad interpretativa, la estructura material y una condición modular que requiere movilizar prácticas materiales de coordinación ecológicamente situadas.

\section{Agradecimientos}

El presente texto fue realizado en el marco de financiamiento de los proyectos: FONDECYT Regular № 1180887 Intereses y Controversias en torno al Agua. Los grupos de interés y la reforma al Código de Aguas y FONDART Nacional № 547745, Línea de Arquitectura, Modalidad de Investigación. 


\section{Bibliografía}

Callon, M. (1984) Some elements of a sociology of translation: domestication of the scallops and the fishermen of St Brieuc Bay. The Sociological Review, 32(1), 196-233. https://doi.org/10.1111/j.1467-954X.1984.tb00113.x

Law, J. (2002). Aircraft stories. Decentering the object in technoscience. Duke University Press.

Park, R.E. (1928) Human migration and the marginal man. American Journal of Sociology, 33(6), 881-893. https://doi.org/10.1086/214592

Simmel, G. (2015). Sociología: estudios sobre las formas de socialización. Fondo de Cultura Económica

Star, S.L. (1989). The structure of ill-structured solutions: boundary objects and heterogeneous distributed problem solving. In L. Gasser; M. Huhns. Distributed Artificial Intelligence, pp. 37-54. https://doi.org/10.1016/b978-1-55860-092-8.50006-x

Thévenot, L. (2016). La acción en plural. Una introducción a la sociología pragmática. Siglo XXI Editores

Vinck, D. (2009). De l'objet intermédiaire à l'objet-frontière: vers la prise en compte du travail d'équipement. Revue d'anthropologie des connaissances, 3(1), 51-72. https://doi.org/10.3917/rac.006.0051

Recibido el 13 Jul 2020

Aceptado el 2 Sep 2020 